\title{
Modeling and Operation of a Bearingless Fixed-Pole Rotor Induction Motor
}

\author{
Xiaoting Ye, Zebin Yang, Jianguo Zhu, and Youguang Guo
}

\begin{abstract}
The rotor currents are induced by both suspension force winding magnetic field and torque winding magnetic field in the traditional bearingless induction motors (BIMs). Due to the currents induced by the suspension force winding, there are errors in the generation of radial suspension forces. To address such problems, a novel BIM with fixed-pole rotor, called as bearingless fixed-pole rotor induction (BFPRI) motor is proposed. The structure of BFPRI motor is first analyzed. And then, the mathematical models of radial suspension forces are deduced. Based on the finite element analysis (FEA), the induced currents and radial suspension forces are also investigated and compared with the traditional BIM. Finally, the prototype motor is built and experimental research is carried out. In this novel motor, only the torque winding magnetic field induces currents in the rotor, which makes the precision of radial suspension forces higher and reduces the complexity of BIM control system. The effectiveness of the proposed BFPRI motor is validated by both simulation and experiments.
\end{abstract}

Index Terms - bearingless induction motor (BIM); fixed-pole rotor; operation principle; radial suspension forces; rotor currents

\section{INTRODUCTION}

A magnetic bearing is a non-contact support between the stator and rotor by electromagnetic forces, which effectively solves the problems such as wear, vibration, noise, lubrication and so on [1]-[3]. However, the motor system supported by magnetic bearings has long axial length, low critical speed and complicated structure, which limits the development of high-speed and high-power magnetic bearing motor. One of the solutions to these shortcomings of magnetic bearing is bearingless motor, which is an integration of the magnetic bearing and the motor, and which produces both the suspension forces and torque in a single motor. Compared with the motor supported by magnetic bearings, the volume is

Manuscript receipt and acceptance dates will be inserted here. This work was supported in part by the National Natural Science Foundation of China under Project 51475214M, in part by the Six Categories Talent Peak of Jiangsu Province under Projects 2014-ZBZZ-017 and 2015-XNYQC-003, in part by the 333 Project of Jiangsu Province under Project BRA2017441, in part by the Zhenjiang Key Research and Development Project under Project GY2016003, and in part by the Priority Academic Program Development of Jiangsu Higher Education Institutions. (Corresponding author: Zebin Yang.)

$X$. Ye and Z. Yang are with the School of Electrical and Information Engineering, Jiangsu University, Zhenjiang 212013, China (e-mail: xiaotingye@163.com; zbyang@ujs.edu.cn).

J. Zhu and Y. Guo are with the School of Electrical, Mechanical and Mechatronic Systems, University of Technology Sydney, Sydney, Australia (e-mail: Jianguo.Zhu@uts.edu.au; Youguang.Guo-1@uts.edu.au).

Color versions of one or more of the figures in this paper are available online at http://ieeexplore.ieee.org.

Digital Object Identifier will be inserted here upon acceptance. smaller, the power density is greater, and the critical speed is higher.

At present, the most widely studied bearingless motors mainly include bearingless permanent magnet synchronous motors, bearingless induction motors (BIMs) and bearingless switched reluctance motors [4]-[7]. In comparison with other motors, BIMs attract a lot of interest owing to its definite advantages, such as reliable operation, easy flux-weakening, low torque ripple and low noise. There are two types of rotors for BIMs, namely wound-rotor and squirrel-cage rotor. Squirrelcage rotor is used far more widely in practical since it possesses the merits of reasonable cost, simple structure and better mechanical robustness. Suspension force winding is added to stator slots of the traditional induction motor, and rotation and suspension of rotor are realized by decoupling two sets of windings of stator. In BIMs, a squirrel-cage rotor induces rotor electromotive force (EMF) and produce rotor inductive currents by both suspension force winding magnetic field and torque winding magnetic field. The rotor currents induced by the suspension force winding magnetic field lead to a response delay and loss in radial suspension forces. In the BIMs of low power and speed, the currents influence of suspension force winding magnetic field is limited. Thus, the induced currents of suspension force winding are negligible to reduce the computational complexity. Nevertheless, in the BIMs of high power and speed, the influence becomes significant as the rotational speed is increased. The errors in radial suspension forces further make the radial suspension forces coupled and the phase margin of suspension system smaller.

Based on the above analysis, a BIM with fixed-pole rotor, namely, bearingless fixed-pole rotor induction (BFPRI) motor is proposed in this paper to address the problems of traditional squirrel-cage rotor BIMs. The rotor structure of this special design, only torque winding magnetic field induces rotor currents, and suspension force winding magnetic field does not, which reduces the complexity of the motor control system. This paper is organized as follows. The basic structure and operation principle of the proposed BFPRI motor will be introduced in Section II. The mathematical models of radial suspension forces are deduced in Section III. Based on the FEA, electromagnetic properties of BFPRI motor are analyzed and studied in detail in Section IV, including rotor currents and radial suspension forces. The experiments of the proposed BFPRI motor were conducted through the given analysis in order to verify characteristics of the motor in Section V. The research results show that rotor currents are not affected by suspension force winding magnetic field, and the errors of ra- 
dial suspension forces decreases. Finally, a conclusion is drawn in Section VI.

\section{MOtor TOPOLOGIES AND PRINCIPLE}

In order to present the comparisons of the traditional BIM and the proposed BFPRI motor, two 3D-FEA models are built for the conventional BIM and BFPRI motor, which have the same stator, electric loading, torque winding and suspension force winding. Fig. 1 shows the stator configuration of the conventional BIM and BFPRI motor, which has 24 slots, 4pole suspension force winding and 2-pole torque winding. The torque winding adopts a three-phase distributed connection. Each phase of torque winding is composed of four windings, which is used to generate rotational torque. The suspension force winding also adopts a three-phase distributed connection in which each phase of suspension force winding is composed of two windings that is used to generate radial suspension forces.

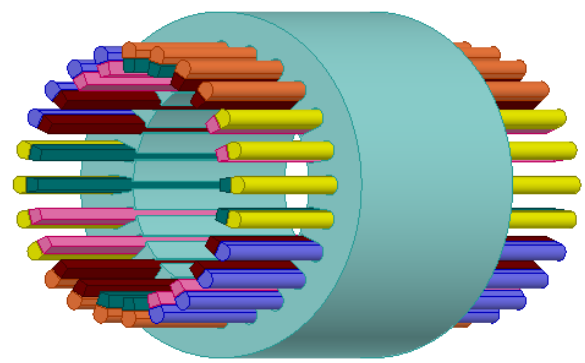

Fig. 1. Stator of the motor

The traditional squirrel-cage rotors for BIMs lead to problems when generating radial suspension force, due to additional 4-pole rotating magnetic field produced in the airgap by the suspension force winding currents. The flux of suspension force winding needs to follow the suspension force in real time and induces EMFs in the rotor which generate rotor current. But the rotor flux cannot produce instantaneous changes, resulting in the delay of the suspension force response. In addition to the response delay, the forces are difficult to orientate due to the alignment of the flux affect by the rotor currents.

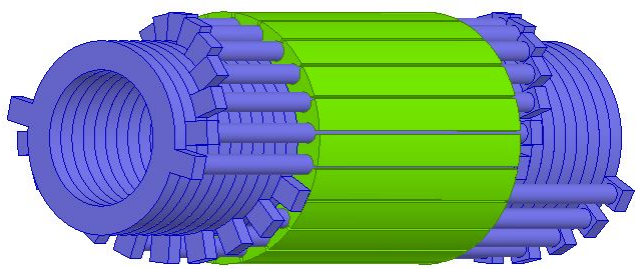

Fig. 2. Fixed-pole rotor of BFPRI motor

To compensate for these deficiencies in the traditional squirrel-cage rotor, the proposed BFPRI motor with fixed-pole rotor is adopted. As can be seen from Fig. 2, a particular structure rotor is employed in the BFPRI motor. The fixed-pole rotor is consisted of 11 sets of special cage rotor bars due to the 22 slots of rotor. These 11 sets of special cage rotor bars need to be isolated from each other. Each set of special cage rotor bars is an independent and complete structure which is made up of two bars and two end-rings. When a 2-pole rotating magnetic field is generated in the airgap by the torque wind- ing, EMFs are induced and currents are produced in the rotor. On the contrast, if a 4-pole rotating magnetic field is applied by the suspension force winding, there is no current in the rotor because the rotor flux linkages between the two bars are cancel each other out, and the sum of the EMFs induced in the special cage rotor bars is zero. Therefore, the proposed BFPRI motor with fixed-pole rotor whose suspension performance is excellent, overcome the demerits of the traditional BIMs.

\section{MATHEMATICAL Models}

For the convenience of analysis, the following simplifications are made in the process of derivation of the mathematical model. The two sets of stator windings are symmetrically distributed in space; the air gap magnetomotive forces (MMFs) generated by the currents have a sinusoidal distribution; higher harmonics, temperature, magnetic saturation, cogging effect, core reluctance, eddy current losses and other effects are neglected. According to the principle of the equivalent magnetic circuit of the motor, the inductance matrix of torque winding and suspension force winding is derived, and the magnetic energy is explained as

$$
W=\frac{1}{2}[\mathrm{i}]^{T}[\mathrm{~L}][\mathrm{i}]
$$

in which $W$ is magnetic energy, [i] is current matrix of torque winding and suspension force winding, $[\mathrm{L}]$ is inductance matrix of torque winding and suspension force winding.

The mathematical model of radial suspension forces can be obtained by the partial derivation with respect to the rotor displacement by electromagnetic energy storage,

$$
\left[\begin{array}{l}
F_{x} \\
F_{y}
\end{array}\right]=\left[\begin{array}{c}
\frac{\partial W}{\partial x} \\
\frac{\partial W}{\partial y}
\end{array}\right]
$$

where $F_{x}$ and $F_{y}$ are the component forces of radial suspension forces on the $x$-axis and $y$-axis directions, respectively.

In order to achieve stable rotor suspension operation, stable and controllable radial suspension forces must be generated in the BIM. The stator slots of the BIM need to embed the torque winding and suspension force winding with a difference in pole pairs of 1 , and angular frequency of the two sets of winding currents are equal. Moreover, for a traditional BIM with 4pole suspension force winding and 2-pole torque winding, three-phase to two-phase transformation is executed to simplify the calculation. Then the calculation of radial suspension forces in a simple expression is written as

$$
\left[\begin{array}{c}
F_{x} \\
F_{y}
\end{array}\right]=M_{z s}\left[\begin{array}{cc}
-\left(i_{z s d}+i_{z r d}\right) & i_{z s q}+i_{z r q} \\
i_{z s q}+i_{z r q} & i_{z s d}+i_{z r d}
\end{array}\right]\left[\begin{array}{c}
i_{s s d}+i_{s r d} \\
i_{s s q}+i_{s r q}
\end{array}\right]
$$

where $M_{\mathrm{zs}}$ is mutual inductance between two windings of the stator, $i_{\text {ssd }}$ and $i_{\text {srd }}$ are suspension force winding $d$-axis current of stator and rotor, respectively, $i_{\text {ssq }}$ and $i_{\text {srq }}$ are suspension force winding $q$-axis current of stator and rotor, respectively, $i_{\text {zsd }}$ and $i_{\text {zrd }}$ are torque winding $d$-axis current of stator and rotor, respectively, and $i_{\text {zsq }}$ and $i_{\text {zrq }}$ are torque winding $q$-axis current of stator and rotor. 
The airgap magnetic field of suspension force winding is generated by the current components of $i_{\text {ssd }}+i_{\text {srd }}$ and $i_{\text {ssq }}+i_{\text {srq }}$ in (3). Furthermore, the airgap magnetic field of torque winding is generated by the current components of $i_{\mathrm{zsd}}+i_{\mathrm{zrd}}$ and $i_{\mathrm{zsq}}+i_{\mathrm{zrq}}$. It can be easily found that radial suspension forces are generated by an interaction of current components of suspension force winding magnetic field and torque winding magnetic field. On account of the influence of suspension force winding current, the induced currents in the rotor leads to the response delay of the radial suspension forces. In other words, complex control system is needed to reduce the influence of suspension force winding on the radial suspension forces.

For a BFPRI motor, suspension force winding currents of rotor $i_{\text {srd }}$ and $i_{\text {srq }}$ are zero, thanks to the structure of fixed-pole rotor. Thus, Equation (3) can be simplified as

$$
\left[\begin{array}{c}
F_{x} \\
F_{y}
\end{array}\right]=M_{z s}\left[\begin{array}{cc}
-\left(i_{z s d}+i_{z r d}\right) & i_{z s q}+i_{z r q} \\
i_{z s q}+i_{z r q} & i_{z s d}+i_{z r d}
\end{array}\right]\left[\begin{array}{c}
i_{s s d} \\
i_{s s q}
\end{array}\right]
$$

Component currents of airgap magnetic field are defined as follows:

$$
i_{z s d}+i_{z r d}=i_{z d}, i_{z s q}+i_{z r q}=i_{z q}
$$

in which $i_{\mathrm{zd}}$ and $i_{\mathrm{zq}}$ are d-axis and q-axis current components of torque winding, respectively.

With the adjustment of suspension force winding currents, the radial suspension forces can be easily controlled to realize successful magnetic suspension.

\section{Electromagnetic Performance COMPARISON}

\section{A. Rotor Currents}

The angular frequencies of suspension force winding currents and torque winding currents are equal. The computation time is given from $0 \mathrm{~s}$ to $0.4 \mathrm{~s}$. The time step size is configured to $0.001 \mathrm{~s}$. The waveforms of rotor induced currents are analyzed under loaded torque condition when the rotor is rotating in the geometric center position.

To analyze the electromagnetic performance and verify the superiority of the proposed motor, three different cases, i.e. only excited by torque winding, only excited by suspension force winding and excited by two sets of winding, are investigated through FEA for BFPRI motor and the traditional BIM, respectively. Fig. 3 shows the rotor currents waveforms of two types of motors under different current excitation. When only the torque winding applied, the rotor currents waveform is shown in Fig. 3(a). It can be found that the value and trends of rotor currents in BFPRI motor and the traditional BIM are almost same. As shown in Fig. 3(b), with the excitation of suspension force winding, the rotor currents of the BFPRI motor is essentially zero, while the currents of the conventional BIM are still sinusoidal waveform changing from $-250 \mathrm{~A}$ to $150 \mathrm{~A}$, by which the airgap magnetic field will be seriously influenced. Moreover, it will delay the suspension forces response and cause errors. In Fig. 3 (c), the torque winding and suspension force winding are both excited. The waveform of the induced current in the traditional BIM appears distorted due to the influence of the rotating 4-pole magnetic field generated by the suspension force winding currents. The induced currents of the BFPRI motor is identical with the ones under torque winding excitation alone. According to this analysis, good suspension performance can be realized by the proposed BFPRI motor.

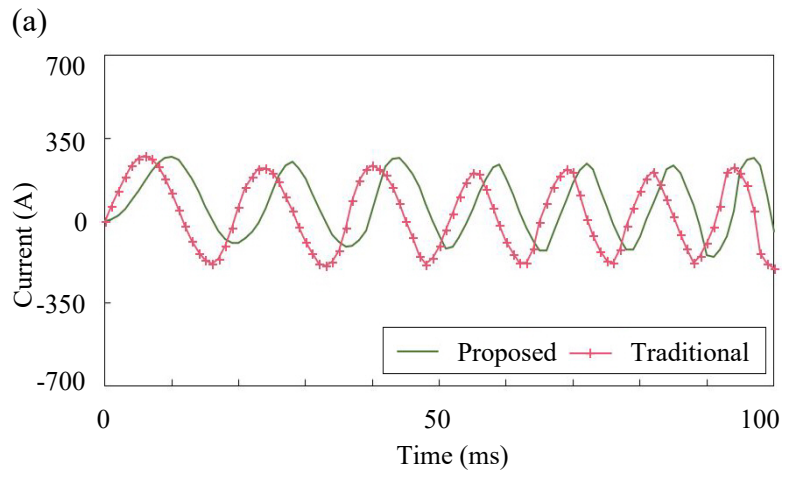

(b)

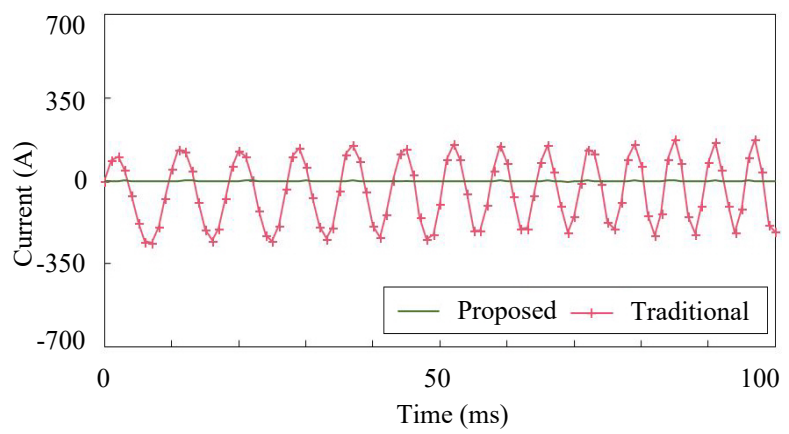

(c)

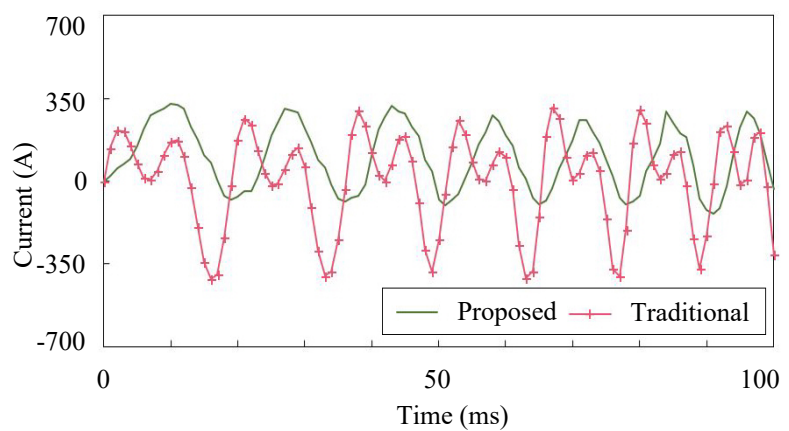

Fig. 3. Rotor currents waveforms of the traditional BIM and BFPRI motor (a) Excited by torque winding (b) Excited by suspension force winding (c) Excited by two sets of windings

\section{B. Radial Suspension Forces}

The radial suspension forces waveforms of the traditional BIM and BFPRI motor are depicted in Fig. 4, respectively. $F_{\mathrm{x}}$ and $F_{\mathrm{y}}$ are the component forces of radial suspension forces on the $x$-axis and $y$-axis directions, respectively. $F_{\mathrm{m}}$ is the resultant forces of radial suspension forces. The average value of radial suspension forces in the conventional BIM and in BFPRI motor are approximately $170 \mathrm{~N}$ and $420 \mathrm{~N}$, respectively, which validate that the rotor currents induced by the suspension force winding magnetic field lead to a loss in radial suspension forces. Besides, it is worthy to note that the waveforms of $F_{\mathrm{x}}$ and $F_{\mathrm{y}}$ has deformation and is no longer a sine wave in the traditional BIM, which further make the large rip- 
ple of resultant forces. In contrast, the radial suspension force components in the $x$ - and $y$-directions are almost sinusoidal for the BFPRI motor. The resultant forces have higher stability. Moreover, the radial suspension forces can be easily controlled by the control system in the BFPRI motor.

(a)

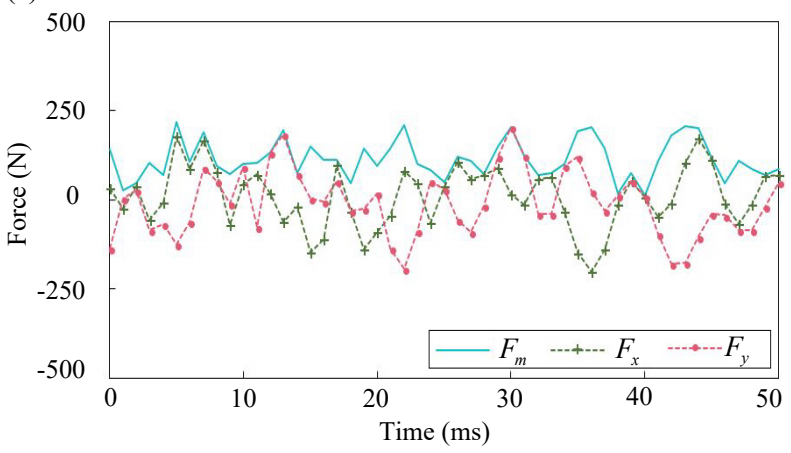

(b)

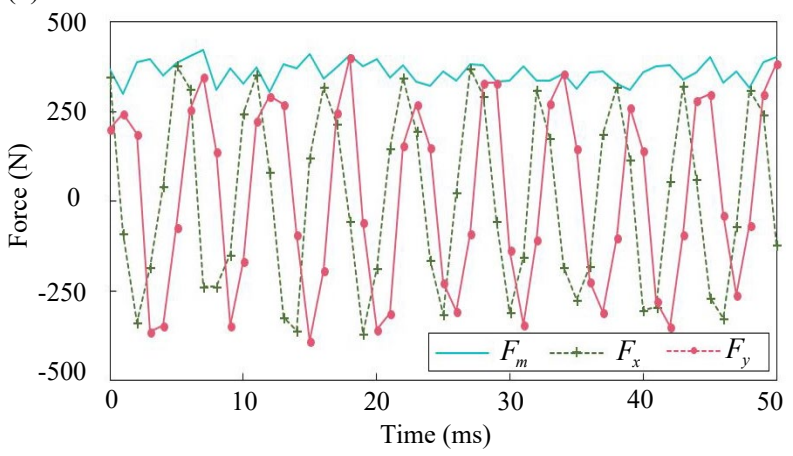

Fig. 4. Radial suspension forces waveforms (a) Traditional BIM (b) BFPRI motor

The FEA results verify aforementioned theory that only torque winding will induce EMFs and currents in the rotor in BFPRI motor.

\section{EXPERIMENTS}

To validate the analysis and performance of the BFPRI motor, an experimental system is set up in the laboratory. A 24slots stator, 11 sets of fixed-pole rotor bars BFPRI motor is built as an experimental prototype motor. The basic parameters of the experimental prototype are as follows: stator outer radius is $77.5 \mathrm{~mm}$, stator inner radius is $49 \mathrm{~mm}$, core length is $105 \mathrm{~mm}$, rotor outer radius is $48.5 \mathrm{~mm}$, rotor inner radius is 18 $\mathrm{mm}$, airgap length is $0.5 \mathrm{~mm}$.

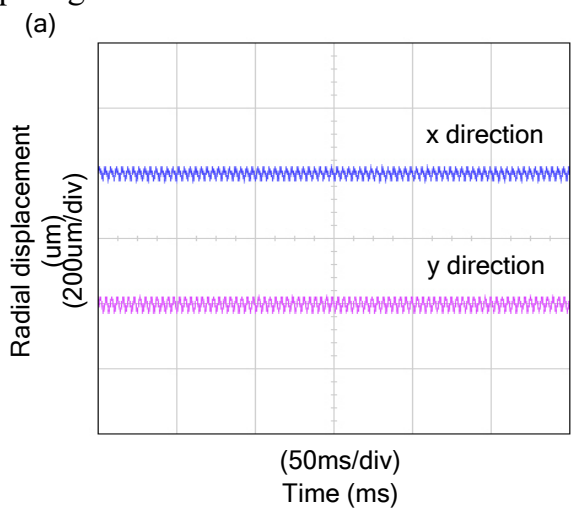

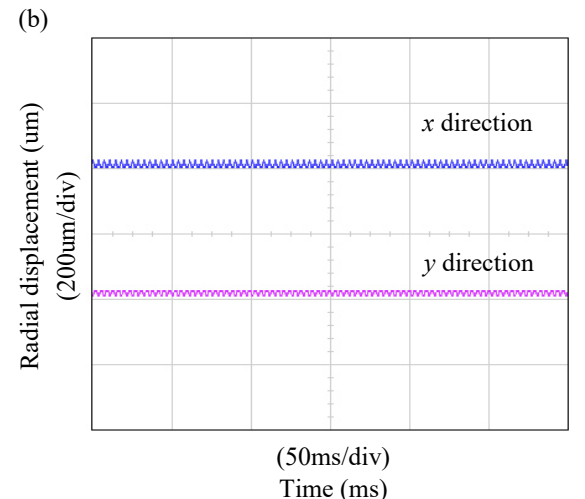

Fig. 5. Experimental result of rotor radial displacement (a) Traditional BIM (b) BFPRI motor

The radial displacement curves in $x$ - and $y$-direction for the traditional BIM and BFPRI motor are illustrated in Fig. 5, respectively. It should be noted from Fig. 5 that the peak to peak values of the displacement fluctuation in the BFPRI motor and the traditional BIM are 70 um and $40 \mathrm{um}$, respectively. Compared with the BFPRI motor and the traditional BIM, the fluctuation range is decreased by $40 \%$, which is much less than the airgap length of the motor. The stable suspension operation is realized, and control precision is improved.

\section{CONCLUSION}

In this paper a 24-slots stator, 11 sets of fixed-pole rotor BFPRI motor is proposed. The basic motor structure, rotor configuration, operation principle and mathematical model are introduced. The electromagnetic performances of the BFPRI motor were predicted through FEA. According to the FEA simulation results and the experiments, the proposed BFPRI motor can realize stable suspension and operation, which improve the control accuracy of suspension forces. The BFPRI motor provides a promising solution to the further improvement of BIM design and performance optimization.

\section{REFERENCES}

[1] S. Xu and J. Sun, "Decoupling structure for heteropolar permanent magnet biased radial magnetic bearing with subsidiary air-gap," IEEE Trans. on Magn., vol. 50, no. 8, pp. 1-8, Aug. 2014.

[2] M. Sparing, M. Hossain, and D. Berger, et al., "Superconducting magnetic bearing as twist element in textile machines," IEEE Trans. Appl. Supercond., vol. 25, no. 3, pp. 1-4, June 2015.

[3] S. Mukoyama et al., "Development of superconducting magnetic bearing for $300 \mathrm{~kW}$ flywheel energy storage system," IEEE Trans. Appl. Supercond., vol. 27, no. 4, pp. 1-4, June 2017.

[4] X. Sun, Z. Shi, L. Chen and Z. Yang, "Internal model control for a bearingless permanent magnet synchronous motor based on inverse system method," IEEE Trans. Energy Convers., vol. 31, no. 4, pp. 1539-1548, Dec. 2016.

[5] Z. Yang, L. Chen, X. Sun, et al., "A bearingless induction motor direct torque control and suspension force control based on sliding mode variable atructure," Math. Problems in Eng., vol. 2017, pp.1-11, 2017, Art. ID 2409179.

[6] Z. Yang, D. Dong, H. Gao, X. Sun, R. Fan, and H. Zhu, "Rotor mass eccentricity vibration compensation control in bearingless induction motor," Adv. Mechanical Eng., vol. 7, no. 1, pp. 1-8, 2015, Art. ID168428.

[7] H. Wang, J. Bao, B. Xue and J. Liu, "Control of suspending force in novel permanent-magnet-biased bearingless switched reluctance motor," IEEE Trans. Ind. Electron., vol. 62, no. 7, pp. 4298-4306, July 2015. 